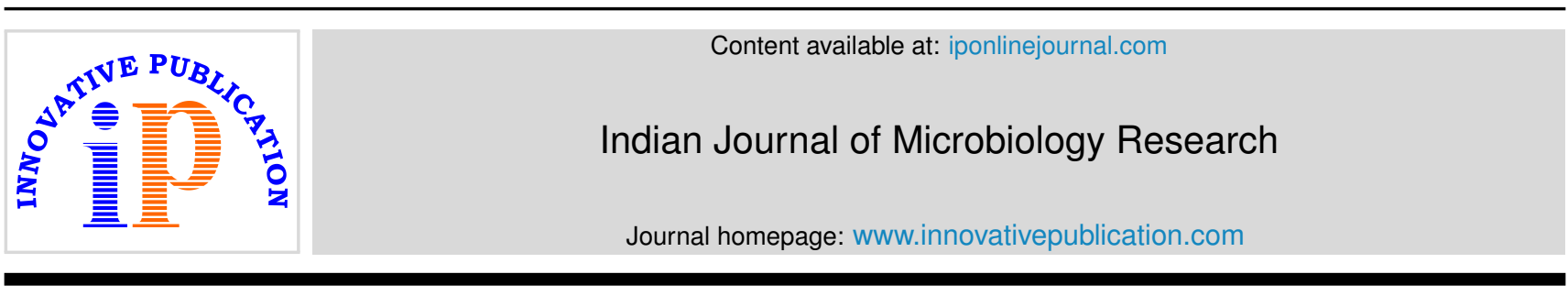

Original Research Article

\title{
Prevalence of non albicans candida species in a tertiary care hospital
}

\author{
Y. Raja Ratna Reddy ${ }^{1}$, M. Nissi Priya ${ }^{1, *}$, B. Ramachandra Reddy ${ }^{1}$, Manisha Singh ${ }^{1}$, \\ Vasanti Kabra ${ }^{1}$ \\ ${ }^{1}$ Dept. of Microbiology, SVS Medical College, Mahbubnagar, Telangana, India
}

\section{A R T I C L E I N F O}

Article history:

Received 26-07-2019

Accepted 04-09-2019

Available online 09-09-2019

\section{Keywords:}

Non albicans Candida

Candida utilis

Fungemia

Virulence

\begin{abstract}
A B S T R A C T
Introduction: Candida species are recognized as the most important fungal pathogens with significant contribution to the morbidity and mortality in critically ill patients. C. albicans is the leading cause of fungal infections until the recent emergence of non albicans Candida (NAC) infections, therefore transforming the current epidemiological trends.

Materials and Methods: The clinical samples received in the microbiology department were cultured on Sabouraud' s dextrose agar followed by identification and sensitivity testing of Candida species using automated VITEK 2-compact (biomerieux) instrument.

Results and Discussion: The present study also shows the prevalence of NAC species with C. utilis being the most significant isolate from neonatal blood cultures. C. utilis is generally considered as an industrial yeast with low virulence, hence occasionally reported from clinical samples. However, their clinical relevance should be interpreted with strong clinical and microbiological correlation.

Conclusion: Surveillance for emerging fungal pathogens is essential to monitor the changing epidemiological trends for controlling the spread of NAC species
\end{abstract}

(C) 2019 Published by Innovative Publication .

\section{Introduction}

Candida species are recognized as the most important fungal pathogens with significant contribution to the morbidity and mortality in critically ill patients. ${ }^{1}$ They are responsible for $9-13 \%$ of blood stream infections in neonates ${ }^{2}$ and a significant proportion of these infections are nosocomial origin. The known risk factors are immunosuppression, use of broad spectrum antibiotics, prematurity and invasive devic es. Although Candida albicans account for vast majority of infections, however, in recent past the emergence of non albicans Candida (NAC) species such as; C. tropicalis, C. parapsilosis, C. krusei, and C. glabrata are being increasingly reported throughout the world. $^{3}$

The transition of Candida species from commensal to potent pathogen is facilitated by number of virulence factors such as; adhesion to host tissue, biofilm formation and

\footnotetext{
* Corresponding author.

E-mail address: nissipriya86@gmail.com (M. N. Priya).
}

secretion of hydrolytic enzymes. ${ }^{4}$ Most of these virulence markers are extensively investigated and recognized as the important attributes of $C$. albicans pathogenicity, however, very little is known about the pathogenic determinants for NAC species. Several of these NAC species are shown to exhibit intrinsic or acquired resistance to commonly used antifungal drugs, therefore increasing their incidence in recent times. Besides, drug resistant NAC species are responsible to cause a significant proportion of blood stream infections with increased mortality in critically ill cases such as cancer patients. ${ }^{5}$

Recently, there has been a change in the epidemiology of Candida infections, characterized by a progressive shift from a preponderance of $C$. albicans to NAC species, consequently having greater impact on the selection of appropriate therapy. In addition, the incidence and distribution of NAC species in our area is currently unknown. Therefore, the present study is highly essential to know the prevalence of NAC species from clinical specimens and antifungal susceptibilities of these isolates is 
currently imperative to guide optimal empirical treatment.

\section{Materials and Methods}

The various types of Candida species isolated from clinical samples received (past one year) for fungal culture and sensitivity in the department of microbiology, SVS medical college were included in the study. Samples were inoculated on two sets of Sabouraud's dextrose agar and incubated at $37^{\circ} \mathrm{C}$ for $24-48 \mathrm{hrs}$. After the incubation period, growth was preliminarily identified as Candida based on the colony morphology and Gram staining. Samples showing significant growth characteristics such as; pure and predominant growth were processed further.

Once they are identified as Candida, further identification and antifungal susceptibility testing of different species of NAC were performed by automated VITEK 2-compact (biomerieux) instrument using VITEK $^{\circledR} 2$ YST and ASTYS08 respectively.

\section{Results}

A total of 96 Candida species were isolated from various clinical samples. Overall, the incidence of NAC species is significantly higher (90) than $C$. albicans (6). The various NAC species isolated includes; $C$. utilis $(79 ; 82.3 \%), C$. tropicalis $(7 ; 7.3 \%)$ and $C$. glabrata $(4 ; 4.2 \%)$, whereas, the incidence rate of $C$. albicans is relatively at low (6.2\%) Table 1 . A vast majority of the NAC species were isolated from blood (77) and urine (8) compared to their incidence in other clinical samples including endotracheal secretions (2), branchoalveolar lavage (1), throat swab (1) and stool (1). However, the isolation of $C$. albicans from clinical specimens is limited to blood (5) and urine (1) only.

Majority of the NAC species (78) were isolated from newborns possibly because of immature defense mechanisms and more invasive devises, therefore, predisposing them to Candida infections. C. utilis is the predominant NAC species (74) isolated in our study especially from neonatal blood cultures. Although, the incidence of $C$. albicans in our study is significantly lower than the former, however, it is the second leading (5) cause of newborn blood stream infections Table 2.

Antifungal susceptibility testing of all the Candida species were performed by automated VITEK 2-compact instrument. All the species were found to be sensitive to the tested antifungal drugs such as; fluconazole, voriconazole, amphotericin, flucytosine and caspofungin ; while drug resistance found in the study appears to be very insignificant (data not shown).

\section{Discussion}

C. utilis is extensively used in the food industry for its ability to perform nonethanolic fermentation reactions for production of acetaldehyde and many other useful organics. ${ }^{6}$ The clinical significance of $C$. utilis is often discounted possibly because of its low virulence, therefore rarely isolated and interpreted from clinical specimens. ${ }^{7}$ Although, infrequent, the opportunity of $C$. utilis being pathogenic has been found in the literature published from different parts of the world.

The incidence and clinical significance of $C$. utilis has been fairly associated to cause variety of infections including; catheter related bloodstream infections in patients with metastatic carcinoma of the bladder, ${ }^{8}$ neonatal candidemia, ${ }^{9}$ chronic urinary tract infection, ${ }^{10}$ fungemia in non -neutropenic patients ${ }^{11}$ and patients with acquired immunodeficiency syndrome. ${ }^{12}$

In our study, $C$. utilis is the most predominant NAC species isolated from neonatal blood stream infections. As most of the species isolated were from inpatient samples, it also indicates the significance of Candida in the etiology of nosocomial infections. The role of environment and/or hospital staff are assumed to be potential source of infection. The significant changes that are occurring in the epidemiology of Candida infections could be due to the recent emergence of NAC species as reported from all over the world. However, the virulence markers that transform NAC species as potential blood stream pathogens is need to understand fully. Otherwise, uncertainty regarding the clinical relevance of an isolate of $C$. utilis will continue to trouble.

\section{Conclusion}

The study strongly reemphasizes the current scenario of changing epidemiology of Candida infections with a predominant shift towards NAC species. Strong clinical correlation and precise microbiological prediction are highly essential while establishing the clinical significance of NAC species to preclude the possibilities of being treated them as commensals in life threatening infections. Further, a stringent surveillance for emerging fungal pathogens is extremely important to monitor the changing trends in incidence, geographical distribution and antifungal susceptibility patterns, so as to limit the spread of NAC species. 
Table 1: Prevalence of Candida species from clinical samples

\begin{tabular}{lllllllll}
\hline Species & Blood & Urine & ET & BAL & Stool & $\begin{array}{l}\text { Throat } \\
\text { swab }\end{array}$ & Total & \% \\
C. utilis & 74 & 3 & 1 & 1 & & & 79 & 7.3 \\
C. tropicalis & & 4 & 1 & & 1 & & 4 & 7.3 \\
C. glabrata & 3 & 1 & & & & 6 & 6.2 \\
C. albicans & 5 & 1 & & & & & 6 \\
\hline
\end{tabular}

ET- endotracheal secretion; BAL- branchoalveolar lavage

Table 2: Age wise distribution of different Candida species

\begin{tabular}{|c|c|c|c|c|c|}
\hline & New born & Up to $20 y r s$ & 21-40yrs & 41-60yrs & Above 60yrs \\
\hline C. utilis & 74 & 2 & 3 & & \\
\hline C. tropicalis & 1 & 2 & 1 & 1 & 2 \\
\hline C. glabrata & 3 & & 1 & & \\
\hline C. albicans & 5 & & 1 & & \\
\hline
\end{tabular}

\section{Acknowledgments}

The authors thank S.V.S Medical College, Telangana, India for their essential support.

\section{Conflict of Interest}

None.

\section{Source of Funding}

None.

\section{References}

1. Pfaller M, Diekema DJ. Epidemiology of invasive candidiasis: a persistent public health problem. Clin Microbiol Rev. 2007;20(1):13363.

2. Benjamin DK, Stoll BJ, Fanaroff AA, Mcdonald SA, Oh W, et al. National Institute of Child Health and Human Development Neonatal Research Network. Neonatal candidiasis among extremely low birth weight infants: Risk factors, mortality rates, and neurodevelopmental outcomes at 18 to 22 months. Pediatrics. 2006;117:84-92.

3. Oberoi JK, Wattal C, Goel N, Raveendran R, Datta S, Prasad K. Nonalbicans Candida species in blood stream infections in a tertiary care hospital at New Delhi, India. Indian J Med Res. 2012;136:997-1003.

4. Sachin K, Ruchi, Santosh. In vitro evaluation of proteinase, phospholipase and haemolysin activities of Candida species isolated from clinical specimens. Int J Med Biomed Res. 2012;1(2):153-7.

5. Slavin MA, Sorrell TC, Marriott D. Candidaemia in adult cancer patients: risks for uconazole-resistant isolates and death. J Antimicrob Chemother. 2010;65:1042-51.

6. Prior B, Kilians S, Lategan P. Growth of Candida utilis on ethanol and isopropanol. Arch Microbiol. 1980;125(1-2):133-6.

7. Holzeshu DL, Chandler FW, Ajello L, Ahearn DG. Evaluation of industrial yeasts for pathogenicity. Sabouraudia. 1979;17(1):71-8.
8. AN GS, Donato AC, B ED, B AV, B LV, et al. Candida utilis catheterrelated blood stream infection. Med Mycol Case Rep. 2014;(6):70-2.

9. Luki-Grli A, Mlinari-Missoni E, Skari I, Vazi-Babi V, Svetec IK. Candida utilis candidaemia in neonatal patients. J Med Microbiol. 2011;60(6):838-41.

10. Hazen KC, Theisz GW, Howell SA. Chronic urinary tract infection due to Candida utilis. J Clin Microbiol. 1999;37(3):824-831.

11. Bougnoux ME, Guehoe E, Potocka AC. Resolutive Candida utilis fungemia in a nonneutropenic patient. $J$ Clin Microbiol. 1993;31(6):1644-5.

12. Alsina A, Mason M, Uphoff RA, Riggsby WS, Becker JM, et al. Catheter-associated Candida utilis fungemia inapatient with acquired immunodeficiency syndrome: species verification with a molecular probe. J Clin Microbiol. 1988;26(4):621-4.

\section{Author biography}

Y. Raja Ratna Reddy Associate Professor

M. Nissi Priya Assistant Professor

B. Ramachandra Reddy Assistant Professor

Manisha Singh Professor

Vasanti Kabra Professor and Head

Cite this article: Reddy YRR, Priya MN, Reddy BR, Singh M, Kabra

V. Prevalence of non albicans candida species in a tertiary care hospital. Indian J Microbiol Res 2019;6(3):277-279. 